\title{
FINITE ELEMENT APPROXIMATIONS OF FLUID-STRUCTURE INTERACTION PROBLEMS: NUMERICAL ANALYSIS OF THREE APPROACHES OF AERODYNAMIC FORCES EVALUATION
}

\author{
Jan Valášek 1,2 and Petr Sváček 1, 2 \\ ${ }^{1}$ Department of Technical Mathematics, Faculty of Mechanical Engineering, \\ Czech Technical University in Prague, Karlovo nám. 13, Praha 2, 121 35, Czech Republic \\ 2 Center of Advanced Aerospace Technology, Czech Technical University in Prague, \\ Jugoslavskych partyzanu 1580, Praha 6, 160 00, Czech Republic
}

\begin{abstract}
The aerodynamic forces calculation (AFC) is an important part of the fluid-structure interaction problem. In order to increase the overall accuracy three possible methods of AFC within finite element framework are presented. The extrapolation from the interior of the fluid domain is the most straightforward method, the local reconstruction method is based on the idea of the local velocity gradient reconstruction by the least square method. The weak reformulation technique uses the weak formulation of the fluid flow problem for expression of the aerodynamic forces integrated over the boundary. The weak reformulation technique is here described for the case of the time dependent domain. The experimental convergence of all mentioned methods for the drag and lift coefficients is tested on the benchmark of static cylinder in cross-flow. Further, the transferred energy is calculated by three presented methods of AFC for the case of moving cylinder.
\end{abstract}

Keywords: Navier-Stokes equations, aerodynamic forces, drag, lift, finite element method.

\section{Introduction}

This study is motivated by a reliable simulation of fluid-structure interaction (FSI) problem having many technical applications like airfoil stability or biomechanical applications like hemodynamics or vocal folds vibrations, see [3], [4]. An important part of the numerical solution of FSI is the aerodynamic force calculation (AFC). Due to a coupled nature of the FSI problem it is highly desirable to implement AFC with higher accuracy. AFC has even higher importance in the cases where flutter regime can emerge and the determination of critical velocity of flutter onset is of the interest like for airfoil stability problem or rotating blades in turbine, see [3]. As a good predictor of flutter occurrence the balance of energy transferred from fluid flow to a vibrating body can be used.

More generally the CFD simulations provide primary variables of velocity and pressure however so called derived variables like aerodynamic force, heat flux or vorticity (i.e. the variables which can be obtained first after postprocessing of primary variables) is quite often the sought quantity. Due to a need of numerical derivation of approximated primary variables a special attention should be paid to their numerical implementation in order to improve the overall accuracy of the numerical scheme and the desired results, particularly for finite volume method or finite element method (FEM) of lower orders. The FEM numerical implementation of aerodynamic force is addressed in this contribution.

It is well known that the direct evaluation of boundary integral with velocity gradient appearing in the aerodynamic forces formula provides usually accuracy of one order lower than the accuracy of velocity approximations, see [5], [6]. Classical method how to increase the accuracy of the AFC offers its weak reformulation, see [8], sometimes called consistent force formulation. The key ingredient is the application of the Green theorem on the $L^{2}$-scalar product of the velocity gradient with the FE test function and consequently using the weak formulation of the problem. The advantage of this method is retaining the accuracy of FE velocity approximation, see [6]. The third presented approach is based on the idea of local reconstruction, see [1]. The velocity gradient at quadrature points are reconstructed with the help of least square method using velocity gradient values from adjacent finite elements. Then the boundary integral computed with reconstructed 
velocity gradient should be of higher accuracy (for sufficiently fine meshes). The comparison of all mentioned approaches are performed on the classical benchmark of cylinder in cross-flow, see [7], and in the end the energy transfer from fluid flow to a body with prescribed vibration is calculated based on the aerodynamic forces.

The mathematical description of the flow fluid problem is given in the first section of this article, in the second section three considered techniques of aerodynamic forces calculation (within the FEM) is explained. Third section contains numerical comparison of drag and lift coefficients computed for static cylinder in cross-flow and calculated the energy transferred from the fluid flow for the case of moving cylinder. The conclusion closes this contribution.

\section{Mathematical description}

Let us consider a two-dimensional stationary laminar flow around a possibly moving rigid obstacle with surface $\Gamma_{\mathrm{W}_{\mathrm{t}}}^{f}$ in a channel with static walls $\Gamma_{\mathrm{Dir}}^{f}$ occupied by a fluid. The time dependence of the fluid domain $\Omega_{t}^{f}$ and other sets are highlighted by lower index $t$. We assume that the domain $\Omega_{t}^{f}$ at any time instant $t$ has Lipschitz continous boundary $\partial \Omega_{t}^{f}$, which consists of the inlet boundary $\Gamma_{\text {In }}^{f}$, the outlet $\Gamma_{\text {Out }}^{f}$, channel walls $\Gamma_{\text {Dir }}^{f}$ and the obstacle interface $\Gamma_{\mathrm{W}_{\mathrm{t}}}^{f}$, i.e. $\partial \Omega_{t}^{f}=\overline{\Gamma_{\mathrm{In}}^{f}} \cup \overline{\Gamma_{\text {Out }}^{f}} \cup \overline{\Gamma_{\text {Dir }}^{f}} \cup \overline{\Gamma_{\mathrm{W}_{\mathrm{t}}}^{f}}$, see Figure 2.

In order to address the time dependent formulation of fluid flow problem the arbitrary LagrangianEulerian (ALE) method is used. The ALE method is based on the diffeomorphic mapping $A_{t}$ from the reference domain $\Omega_{\text {ref }}^{f}:=\Omega^{f}(t=0)$ on the domain $\Omega_{t}^{f}$ which is assumed to exist in any time instant $t$. The mapping $A_{t}$ is required to have continuous time derivative, i.e. $\frac{\partial A_{t}}{\partial t} \in C\left(\Omega_{\text {ref }}^{f}\right)$, and to map all reference boundaries to the location of boundaries at $t \in(0, \mathrm{~T})$, i.e. $A_{t}\left(\partial \Omega_{\mathrm{ref}}^{f}\right)=\partial \Omega_{t}^{f}$ and particularly $A_{t}\left(\Gamma_{\mathrm{W}_{\text {ref }}}^{f}\right)=\Gamma_{\mathrm{W}_{\mathrm{t}}}^{f}$ with notation $\Gamma_{\mathrm{W}_{\mathrm{ref}}}^{f}:=\Gamma_{\mathrm{W}}^{f}(t=0)$. Then the ALE domain velocity is defined by

$$
\mathbf{w}_{D}(x, t)=\frac{\partial}{\partial t} A_{t}(X), \quad t \in(0, \mathrm{~T}), x=A_{t}(X) \in \Omega_{t}^{f}
$$

Then ALE derivative is introduced as the time derivative with respect to a fixed point $X \in \Omega_{\text {ref }}^{f}$

$$
\frac{D^{A}}{D t} f(x, t)=\frac{d f}{d t}\left(A_{t}(X), t\right)=\frac{\partial f}{\partial t}(x, t)+\mathbf{w}_{D}(x, t) \cdot \nabla f(x, t) .
$$

The practical construction of ALE mapping $A_{t}$ is described in [4] or [9].

\subsection{Fluid flow}

The considered viscous and incompressible fluid flow model in time dependent domain $\Omega_{t}^{f}$ is given by the Navier-Stokes equations in the ALE formulation

$$
\frac{D^{A} \mathbf{v}}{D t}+\left(\left(\mathbf{v}-\mathbf{w}_{D}\right) \cdot \nabla\right) \mathbf{v}-\nu^{f} \Delta \mathbf{v}+\nabla p=\mathbf{0}, \quad \operatorname{div} \mathbf{v}=0
$$

where vector $\mathbf{v}(x, t)$ denotes the fluid velocity, scalar $p(x, t)$ is the kinematic pressure and the kinematic fluid viscosity is $\nu^{f}$, see [4]. It can be shown that the diffusive and pressure gradient terms of the first equation (3) multiplied by the constant fluid density $\rho^{f}$ can be written as the divergence of the fluid stress tensor $\boldsymbol{\sigma}^{f}=\left(\sigma_{i j}^{f}\right)$ having components

$$
\sigma_{i j}^{f}=\rho^{f}\left(-p \delta_{i j}+\nu^{f}\left(\frac{\partial v_{i}}{\partial x_{j}}+\frac{\partial v_{j}}{\partial x_{i}}\right)\right),
$$


see [5].

The problem (3) is equipped the zero initial condition and the following boundary conditions
a)
$\mathbf{v}(x, t)=\mathbf{v}_{D}(x)$
for $x \in \Gamma_{\operatorname{In}}^{f}$,
b)
$\mathbf{v}(x, t)=\mathbf{0}$
for $x \in \Gamma_{\text {Dir }}^{f}$,
c)
$\mathbf{v}(x, t)=\mathbf{w}_{D}(x, t)$
for $x \in \Gamma_{\mathrm{W}_{\mathrm{t}}}^{f}$,
d)
$p(x, t) \mathbf{n}^{f}-\nu^{f} \frac{\partial \mathbf{v}}{\partial \mathbf{n}^{f}}(x, t)=-\frac{1}{2} \mathbf{v}\left(\mathbf{v} \cdot \mathbf{n}^{f}\right)^{-}$
for $x \in \Gamma_{\text {Out }}^{f}$,

where $\mathbf{v}_{D}(x)$ is a given inlet profile, vector $\mathbf{n}^{f}$ is the unit outer normal to the boundary $\partial \Omega_{t}^{f}$ and symbol $(\alpha)^{-}$denotes $\min (\alpha, 0)$. Condition $(5 \mathrm{~d})$ is so called directional do-nothing boundary condition, see [2].

\subsection{Drag and lift coefficients}

The aerodynamic force density $\mathbf{q}=\left(q_{i}\right)\left[\mathrm{N} / \mathrm{m}^{2}\right]$ can be defined at any point $x$ of obstacle interface $\Gamma_{\mathrm{W}_{\mathrm{t}}}^{f}$ by

$$
q_{i}(x, t)=-\sigma_{i j}^{f}(x, t) n_{j}(x, t)=\rho^{f}\left(p \delta_{i j}-\nu^{f}\left(\frac{\partial v_{i}}{\partial x_{j}}+\frac{\partial v_{j}}{\partial x_{i}}\right)\right) n_{j}(x, t),
$$

where the (summation) convection for $j=1,2$ is used (2D case is considered).

Further under assumption of the flow direction oriented along the $x$-axis and the gravity effects acting against the $y$-axis the drag $D[N / m]$ and the lift $L[N / m]$ exerted by fluid on the obstacle are given by

$$
D=-\int_{\Gamma_{\mathrm{W}}^{f}} \sum_{j=1}^{2} \sigma_{1 j}^{f} n_{j} \mathrm{~d} S, \quad L=-\int_{\Gamma_{\mathrm{W}}^{f}} \sum_{j=1}^{2} \sigma_{2 j}^{f} n_{j} \mathrm{~d} S,
$$

i.e. drag and lift represent the sum of the first and the second component of aerodynamic forces, respectively, see e.g. [6]. Considering a characteristic velocity $V_{\infty}$ the lift and the drag is nondimensionalized by introduction of the lift and the drag coefficients, respectively, as follows

$$
c_{D}=\frac{D}{\frac{1}{2} \rho^{f} V_{\infty}^{2} A}, \quad c_{L}=\frac{L}{\frac{1}{2} \rho^{f} V_{\infty}^{2} A},
$$

where $A$ is in general the cross-section area of the obstacle in the direction $V_{\infty}$, see e.g. [8], here for the $2 \mathrm{D}$ case $A[m]$ denotes cross-section curve diameter of the obstacle in the direction $V_{\infty}$.

\section{Numerical model}

For the numerical solution of fluid flow problem the FEM is used. Nevertheless first, problem (3) is discretized in time. The time interval [0,T] is equidistantly divided in $N$ time steps with length $\Delta t$ given by $\Delta t=\frac{T}{N}$, i.e. time instant $t_{n}$ is $t_{n}=n \Delta t, n=0,1, \ldots, N$. Further the numerical approximations at the $n$-th time level are denoted by $\mathbf{v}^{n} \approx \mathbf{v}\left(t_{n}\right), p^{n} \approx p\left(t_{n}\right)$, etc.

The ALE derivative is approximated by the BDF2 formula as

$$
\frac{D^{A} \mathbf{v}}{D t}\left(t_{n+1}\right) \approx \frac{3 \mathbf{v}^{n+1}-4 \overline{\mathbf{v}}^{n}+\overline{\mathbf{v}}^{n-1}}{2 \Delta t},
$$

where for a fixed time instant $t_{n+1}$ we denote $\overline{\mathbf{v}}^{i}(x)=\mathbf{v}^{i}(\tilde{x})$ for $\tilde{x}=A_{t_{i}}\left(A_{t_{n+1}}^{-1}(x)\right), i \in\{n-1, n\}$ and $x \in \Omega_{t_{n+1}}^{f}$. Further, the spatial discretization at a fixed time instant $t_{n+1}$ is described and thus the upper (time) index $n+1$ is omitted (where possible), i.e. we denote $\mathbf{v}:=\mathbf{v}^{n+1}, \Omega^{f}:=\Omega_{t_{n+1}}^{f}$, etc.

For the purpose of FEM the fluid flow problem is weakly reformulated. The standard procedure with the help of time discretization (9) leads to the problem: 
Find a pair $V=(\mathbf{v}, p) \in \mathbf{H}^{1}\left(\Omega^{f}\right) \times L^{2}\left(\Omega^{f}\right)$ such, that $V$ satisfies boundary conditions $(5 \mathrm{a}, \mathrm{b}, \mathrm{c})$ and equation

$$
a(V, V, \Phi)=f(V),
$$

is satisfied for any test function $\Phi=(\boldsymbol{\varphi}, q)$ from space $\mathbf{X} \times M$. The space $\mathbf{X}$ is introduced as $\mathbf{X}=\left\{\mathbf{f} \in \mathbf{H}^{1}\left(\Omega^{f}\right) \mid \mathbf{f}=\mathbf{0}\right.$ on $\left.\Gamma_{\text {In }}^{f} \cup \Gamma_{\text {Dir }}^{f} \cup \Gamma_{\mathrm{W}}^{f}\right\}$ and $M=L^{2}\left(\Omega^{f}\right)$.

The trilinear form $a(\cdot, \cdot, \cdot)$ is defined as

$$
\begin{aligned}
a\left(V^{*}, V, \Phi\right) & =\left(\frac{3 \mathbf{v}}{2 \Delta t}, \boldsymbol{\varphi}\right)_{\Omega^{f}}+\nu^{f}(\nabla \mathbf{v}, \nabla \boldsymbol{\varphi})_{\Omega^{f}}-(p, \operatorname{div} \boldsymbol{\varphi})_{\Omega^{f}}+(q, \operatorname{div} \mathbf{v})_{\Omega^{f}}+ \\
& +\frac{1}{2}\left(\left(\left(\left(\mathbf{v}^{*}-2 \mathbf{w}_{D}\right) \cdot \nabla\right) \mathbf{v}, \boldsymbol{\varphi}\right)_{\Omega^{f}}-\left(\left(\mathbf{v}^{*} \cdot \nabla\right) \boldsymbol{\varphi}, \mathbf{v}\right)_{\Omega^{f}}+\left(\left(\mathbf{v}^{*} \cdot \mathbf{n}\right)^{+} \mathbf{v}, \boldsymbol{\varphi}\right)_{\Gamma_{\text {Out }}^{f}}\right)
\end{aligned}
$$

and a linear functional $f(\cdot)$

$$
f(\Phi)=\left(\frac{4 \overline{\mathbf{v}}^{n}-\overline{\mathbf{v}}^{n-1}}{2 \Delta t}, \varphi\right)_{\Omega^{f}},
$$

where brackets $(\cdot, \cdot)_{D}$ stands for the scalar product in space $L^{2}(D)$ or $\mathbf{L}^{2}(D)$. The convection term is considered in the skew-symmetric form (see [9]), which motivates the specification of the directional do-nothing boundary condition, see [2].

\subsection{Aerodynamic force calculation}

The aerodynamic forces acting on the obstacle and characterized by the lift and the drag coefficients needs to be evaluated. Three applicable methods of the aerodynamic forces evaluation are described. In what follows we denote the regular triangulation of fluid domain $\Omega_{\text {ref }}^{f}$ by $\mathcal{T}_{h}^{f}$.

\subsubsection{Extrapolation from the interior of the fluid domain}

The first approach of the aerodynamic forces evaluation is to extrapolate the aerodynamic quantities from the interior of the fluid domain onto boundary $\Gamma_{\mathrm{W}}^{f}$ and then use these extrapolated values to approximate aerodynamic forces $\mathbf{q}$ given by (6). During drag and lift computation boundary integrals (7) is to be evaluated. For any edge $E$ from the interface the components of the fluid stress tensor are extrapolated from the fluid mesh element $K_{E}$ adjacent to the edge $E$. The boundary integrals are then approximated with the help of a numerical quadrature formula. Nevertheless using the extrapolated values of velocity gradient theoretically decreases the optimal order of convergence, see [5].

\subsubsection{Local reconstruction technique}

The second possibility is the application of the local reconstruction technique introduced by Babuška, see e.g. [1], which can possibly increase the accuracy of aerodynamic forces computation in comparison with the previous approach. The idea is based on the reconstruction of the FE approximation of velocity gradient, i.e. $\nabla \mathbf{v}_{h} \in \mathbb{P}_{k-1}$ is reconstructed to $\nabla \mathbf{v}_{\text {reconstr }} \in \mathbb{P}_{k}$, where $\mathbb{P}_{k}$ is the space of polynomials of degree less or equal $k$ used for $\mathrm{FE}$ velocity approximation. In our case $\nabla \mathbf{v}_{\text {reconstr }}$ is to be evaluated only at quadrature points located at the boundary $\Gamma_{\mathrm{W}}^{f}$. The procedure is following:

To reconstruct the gradient of velocity $\mathbf{v}_{h}$ at a vertex $A$ located on $\Gamma_{\mathrm{W}}^{f}$, the local patch of elements $\mathcal{P}_{A}$, see Figure 1, is used. The local patch $\mathcal{P}_{A}$ is composed of all triangles $K \in \mathcal{T}_{h}^{f}$ being neighbour of the vertex $A$. If patch $\mathcal{P}_{A}$ consists of 2 elements the help vertex at the end of edge given by the intersection of these two triangles is used instead of vertex $A$ for construction of patch $\mathcal{P}_{A}$.

The value of $\nabla \mathbf{v}_{\text {reconstr }}(A)$ is computed using the least square method for fitting data $\nabla \mathbf{v}_{h}\left(S_{K}\right)$ for all $K \in \mathcal{P}_{A}$ where $S_{K}$ is the center of the triangle $K$, see [1].

The local reconstruction procedure is used only for the computation of velocity gradients as the pressure is in in our case chosen as continuous function. Otherwise the same procedure could be 

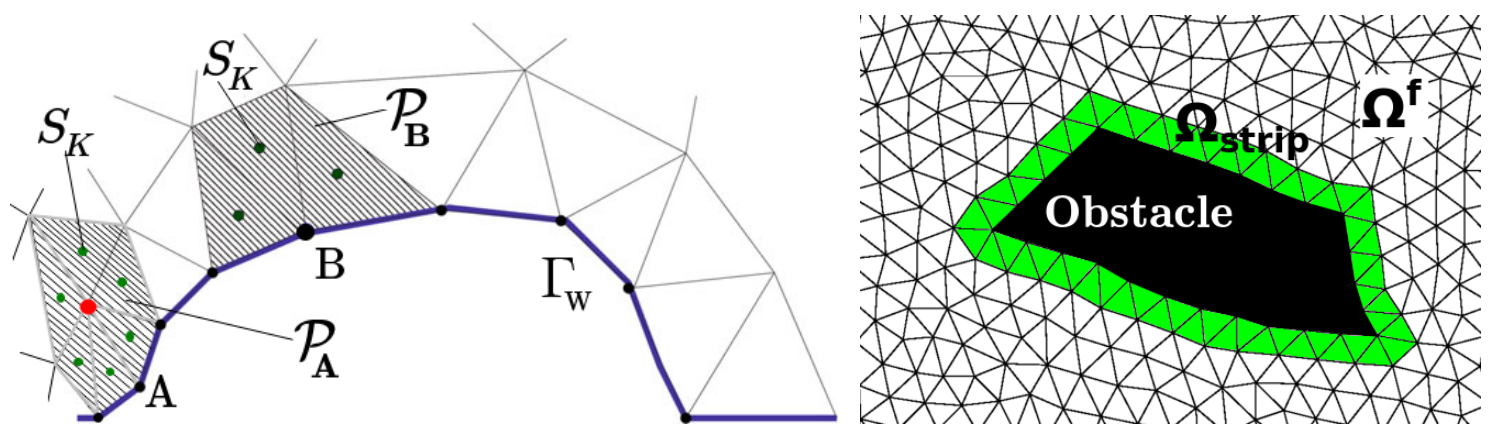

Figure 1: Left: Example of two local patches. The local patch of point $A$ consists of six triangles (a help red point is used for construction of $\mathcal{P}_{A}$ ), while the local patch of point $B$ is constituted by three adjacent triangles. The centres of triangles $S_{K}$ in local patches are denoted by green dots. Right: Scheme of the obstacle (gray) surrounded by (white) fluid domain $\Omega^{f}$ with subdomain $\Omega_{\text {strip }} \subset \Omega^{f}$ marked by green.

used also for $p \in \mathbb{P}_{0}$. The evaluation of the drag and the lift is then done with a numerical quadrature, similarly as above. It provides theoretically higher accuracy compared to the extrapolation procedure, see [1], [5].

\subsubsection{Weak reformulation of aerodynamic forces}

The third possibility how to calculate the aerodynamic forces is to use their weak reformulation. Let us consider fluid domain $\Omega_{\mathrm{t}}^{f}$ with the obstacle interface $\Gamma_{\mathrm{W}_{\mathrm{t}}}^{f}$, see Figure 1 right. We denote one-layer strip of fluid elements around the interface as $\Omega_{\text {strip }}=A_{t_{n+1}}\left(\mathcal{P}_{\Gamma_{W}}\right)$, where the patch $\mathcal{P}_{\Gamma_{W}}$ is given as $\mathcal{P}_{\Gamma_{W}}=\bigcup\left\{K \in \mathcal{T}_{h}^{f} \mid K \cap \Gamma_{\mathrm{W}_{\text {ref }}} \neq \emptyset\right\}$. Next we consider vector function $\varphi_{W} \in \mathbf{H}^{1}\left(\Omega_{\mathrm{t}_{\mathrm{n}+1}}^{f}\right)$ with components $\varphi_{W}=\left(\varphi_{W}^{1}, \varphi_{W}^{2}\right)$ equal to one on boundary $\Gamma_{\mathrm{W}_{\mathrm{t}_{\mathrm{n}+1}}}$ and outside domain $\Omega_{\text {strip }}$ are zero, see [4], i.e. for $j=1,2$

$$
\varphi_{W}^{j}(x)=1 \quad \text { for } x \in \Gamma_{\mathrm{W}}^{f}, \quad \varphi_{W}^{j}(x)=0 \quad \text { for } x \in \Omega^{f} \backslash \Omega_{\text {strip }} .
$$

Further, the first equation of (3) multiplied by the constant fluid density $\rho^{f}$ and by the function $\boldsymbol{\varphi}_{W}$, integrated over the domain $\Omega_{\text {strip }}$ together with application of the Green's theorem can be rewritten as

$$
\left(\boldsymbol{\sigma}^{f} \mathbf{n}^{f}, \boldsymbol{\varphi}_{W}\right)_{\Gamma_{\mathrm{w}_{\mathrm{t}_{\mathrm{n}+1}}}}=\rho^{f}\left(\frac{D^{A} \mathbf{v}}{D t}+\left(\left(\mathbf{v}-\mathbf{w}_{D}\right) \cdot \nabla\right) \mathbf{v}, \boldsymbol{\varphi}_{W}\right)_{\Omega_{\text {strip }}}+\left(\boldsymbol{\sigma}^{f}, \nabla \boldsymbol{\varphi}_{W}\right)_{\Omega_{\text {strip }}}
$$

Using the BDF2 formula on the ALE derivative and the application of spatial discretization, i.e. replacement of $\mathbf{v}, p, \mathbf{w}_{D}$ by the $\mathrm{FE}$ approximations $\mathbf{v}_{h}, p_{h}, \mathbf{w}_{D, h}$ leads to

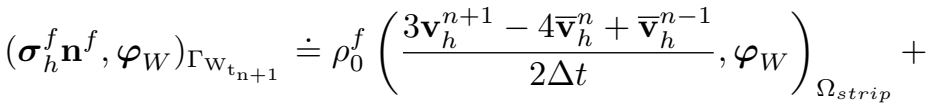

$$
\begin{aligned}
& +\left(\rho_{0}^{f}\left(\left(\mathbf{v}_{h}^{n+1}-\mathbf{w}_{D, h}^{n+1}\right) \cdot \nabla\right) \mathbf{v}_{h}^{n+1}, \boldsymbol{\varphi}_{W}\right)_{\Omega_{\text {strip }}}+\mu^{f}\left(\nabla \mathbf{v}_{h}^{n+1}, \nabla \boldsymbol{\varphi}_{W}\right)_{\Omega_{s t r i p}} \rho_{0}^{f}\left(p_{h}^{n+1}, \operatorname{div} \boldsymbol{\varphi}_{W}\right)_{\Omega_{\text {strip }}},
\end{aligned}
$$

where the last two terms are obtained by definition (6) (in the case of incompressible fluid flow) and $p_{h}^{n+1}$ is the approximation of the kinematic pressure at $t_{n+1}$.

On the other hand formula (6) multiplied by the function $\varphi_{W}$ and integrated over the boundary $\Gamma_{\mathrm{W}_{\mathrm{t}_{\mathrm{n}+1}}}^{f}$ reads

$$
\left(\mathbf{q}, \boldsymbol{\varphi}_{W}\right)_{\Gamma_{\mathrm{W}_{\mathrm{t}_{\mathrm{n}+1}}}}=\left(-\boldsymbol{\sigma}^{f} \mathbf{n}^{f}, \boldsymbol{\varphi}_{W}\right)_{\Gamma_{\mathrm{W}_{\mathrm{t}_{\mathrm{n}+1}}}},
$$

what closes the formulation. In practical implementation the function $\varphi_{W}$ is written with the aid of the Lagrange FE basis consisting of functions $\varphi_{W, k}$, i.e. the same basis functions as used for the velocity discretization of the fluid flow problem on boundary $\Gamma_{\mathrm{W}_{\mathrm{t}}}$. 
Equation (15) offers the another variant how to compute the aerodynamic forces, the drag and lift approximations are the direct consequence of (15). According to analysis [6] this procedure provides to the drag and the lift the same order of accuracy as the original velocity FE approximation under additional regularity assumptions.

\section{$3 \quad$ Numerical results}

All numerical simulations were realized using P1-bubble/P1 elements with parameters set to $\rho^{f}=1.0 \mathrm{~kg} / \mathrm{m}^{3}$ and $\nu^{f}=10^{-3} \mathrm{~m}^{2} / \mathrm{s}$. The convergence of the drag and the lift coefficients for different aerodynamic forces computation procedures is shown for a simple benchmark and then the transferred energy is calculated for a moving obstacle setup.

\section{$3.1 \quad$ Geometry}

The domain $\Omega_{\text {ref }}^{f}$ representing a cylinder in cross-flow is overtaken from paper [6], see Figure 2 . The triangulation of domain $\Omega^{f}$ is performed with the help of concentric circles around the cylinder as in [6]. Then the mesh is multiple times refined by edge splitting leading to the mesh hierarchy, where the finest mesh with label 5 has 123864 nodes (863624 unknowns).

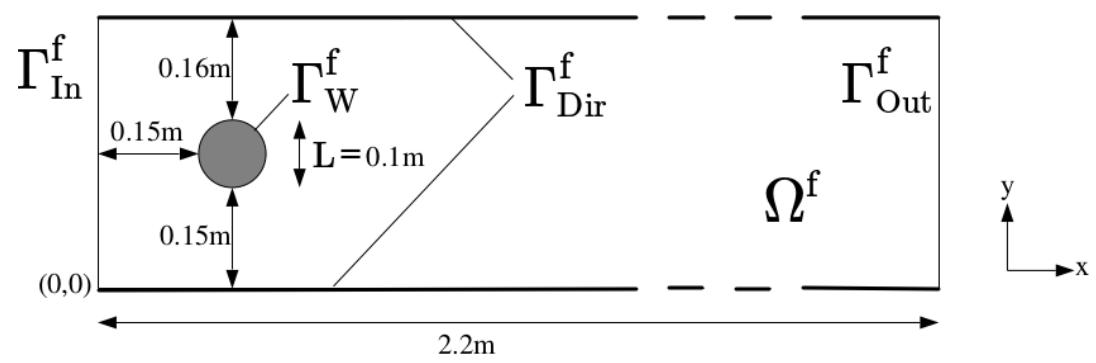

Figure 2: Cylinder obstacle in the middle of the channel together with dimensions. There are denoted boundaries: inlet $\Gamma_{\mathrm{In}}^{f}$, outlet $\Gamma_{\text {Out }}^{f}$, walls $\Gamma_{\mathrm{Dir}}^{f}$ and interface $\Gamma_{\mathrm{W}}^{f}$. Parameter $L$ denotes cylinder diameter.

\subsection{Drag and lift}

The inlet velocity $\mathbf{v}_{D}$ has parabolic profile with mean value $\overline{\mathbf{v}}=0.2 \mathrm{~m} / \mathrm{s}$, i.e. $V_{\infty}=\overline{\mathbf{v}}$, see [7], [6]. Considering cylinder diameter $L=0.1 \mathrm{~m}$ corresponds to Reynolds number $\operatorname{Re}=20$. The upper indices $e x, l r$ and $w f$ are used for values obtained by extrapolation procedure (EX), local reconstruction (LR) technique and weak reformulation (WF) method, respectively.

Tables 1 and 2 show the convergence of the drag and the lift coefficients, respectively. In both cases the coefficients converge to vinicity of the benchmark values: $c_{D}=5.579333, c_{L}=0.010627$ (P1-P0 FE simulation with $6 \mathrm{M}$ unknowns, [6]), $c_{D}=5.5902, c_{L}=0.0108$ (FE simulation $14 \mathrm{~b}$ with Q1-Q0 having 30M unknowns, [7]). The experimental order of convergence EOC is defined as

$$
\operatorname{EOC}\left(c_{X, h}\right)=\log \left(\frac{\left|c_{X, 2 h}-c_{X, h}\right|}{\left|c_{X, h}-c_{X, h / 2}\right|}\right) \log \frac{1}{2},
$$

where $c_{X, h}$ denotes the drag or the lift coefficient computed on mesh with the characteristic element dimension $h$. The EX procedure is the least accurate method of approximately first order accuracy, the LR technique is better reaching EOC around 1.5, while the order EOC for WF method reaches the significantly higher value of circa 2.5 . This is in a relatively good correspondence with analysis [6]. Similarly as in [8] the drag coefficient $c_{D, h}^{e x}$ approaches limit value from below whereas coefficient $c_{D, h}^{w f}$ converges to tabulated values from above. The numerical calculation of lift coefficients has similar behaviour nevertheless with slightly decreased $E O C$ in all cases. Figure 3 (left) depicts aerodynamic forces computed for the considered benchmark on mesh label 4. 


\begin{tabular}{|l|ll|ll|ll|}
\hline Mesh & $c_{D, h}^{e x}$ & $E O C$ & $c_{D, h}^{l r}$ & $E O C$ & $c_{D, h}^{w f}$ & $E O C$ \\
\hline 1 & 5.346845 & & 5.318822 & & 5.593626 & \\
2 & 5.490040 & 1.533 & 5.501330 & 1.812 & 5.582267 & 2.324 \\
3 & 5.539511 & 1.240 & 5.553306 & 1.672 & 5.579999 & 2.574 \\
4 & 5.560453 & 1.100 & 5.569613 & 1.493 & 5.579618 & 2.508 \\
5 & 5.570223 & & 5.575406 & & 5.579551 & \\
\hline
\end{tabular}

Table 1: Comparison of the drag coefficients together with experimental orders of convergence.

\begin{tabular}{|l|ll|ll|ll|}
\hline Mesh & $c_{L, h}^{e x}$ & $E O C$ & $c_{L, h}^{l r}$ & $E O C$ & $c_{L, h}^{w f}$ & $E O C$ \\
\hline 1 & 0.014397 & & 0.030466 & & 0.013905 & \\
2 & 0.011776 & 1.878 & 0.013589 & 2.359 & 0.011374 & 2.151 \\
3 & 0.011063 & 1.483 & 0.010300 & 3.362 & 0.010804 & 2.036 \\
4 & 0.010808 & 1.294 & 0.009980 & 0.715 & 0.010665 & 1.990 \\
5 & 0.010704 & & 0.010175 & & 0.010630 & \\
\hline \hline
\end{tabular}

Table 2: Comparison of the lift coefficients together with experimental orders of convergence.

\subsection{Transferred energy}

A transient computation with a moving cylinder is now presented. The cylinder motion is considered up and down within time interval $t \in[0, \mathrm{~T}]$, i.e. it is prescribed by

$$
u_{1}(x, y)=0, \quad u_{2}(x, y)=C \sin (2 \pi F t), \quad t \in[0, T]
$$

where $\mathbf{u}=\left(u_{1}, u_{2}\right)$ is the cylinder displacement, frequency $F$ is equal to $F=2 \mathrm{~Hz}$ and coefficient $C=-0.08$. The time step is chosen $\Delta t=0.001 \mathrm{~s}$ and the simulation covers four periods of cylinder motion using mesh label 3 with 55314 unknowns.

Figure 3 (right) shows time variation of $c_{D, h}$ for three aforementioned approaches of the drag computation. The coefficients $c_{D, h}^{e x}$ and $c_{D, h}^{l r}$ differ only little against $c_{D, h}^{w f}$ with the biggest difference of $1.6 \%$.
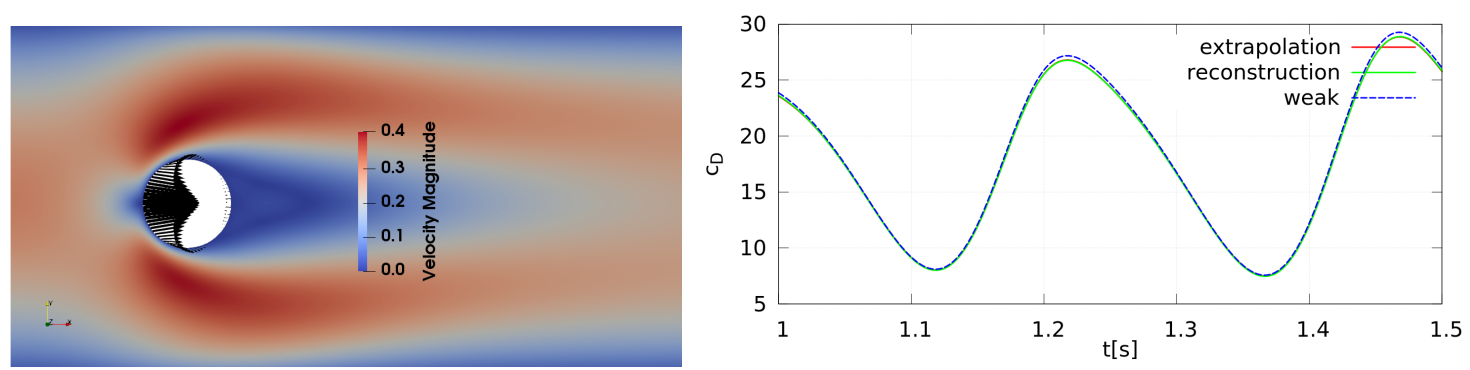

Figure 3: Left: Aerodynamic forces computed by the WF method for the static case with the velocity magnitude plotted on the background. Right: Drag coefficient for cases EX, LR, WF shown in the third period of the cylinder motion.

The rate of energy transfer $\dot{E}_{\text {transf }}$ (power) for the case of a moving surface submerged in fluid flow is given by

$$
\dot{E}_{\text {transf }}=\int_{\Gamma_{\mathrm{W}_{\mathrm{t}}}^{f}} v_{i} \sigma_{i j}^{f}\left(-n_{j}^{f}\right) d S=\int_{\Gamma_{\mathrm{W}_{\mathrm{t}}}^{f}} v_{i} q_{i} d S,
$$

and the transferred energy, described by the cumulative transfer function $E_{\text {cumul }}$, is accumulated power $E_{\text {transf }}$ over the whole time period, see [10],

$$
E_{\text {cumul }}(t)=\int_{0}^{t} \dot{E}_{\text {transf }}(\tau) \mathrm{d} \tau .
$$


Figure 4 depicts a time behaviour of functions $\dot{E}_{\text {transf }}, E_{\text {cumul }}$ for all three considered variants of AFC. The differences are insignificant on the first look, reaching in peak for power $\dot{E}_{\text {transf }}$ circa $1.8 \%$, nevertheless they can accumulate in time. The differences of function $E_{\text {cumul }}$ for cases $E X$ and $W F$ are approximately $0.5 \%$ per period, while the case $L R$ lies in the middle of these two extremes. The function $E_{\text {cumul }}$ is in all cases gradually decreasing, i.e. energy is transferred from obstacle to the fluid flow, what is an expected behaviour for the prescribed motion of the cylinder.
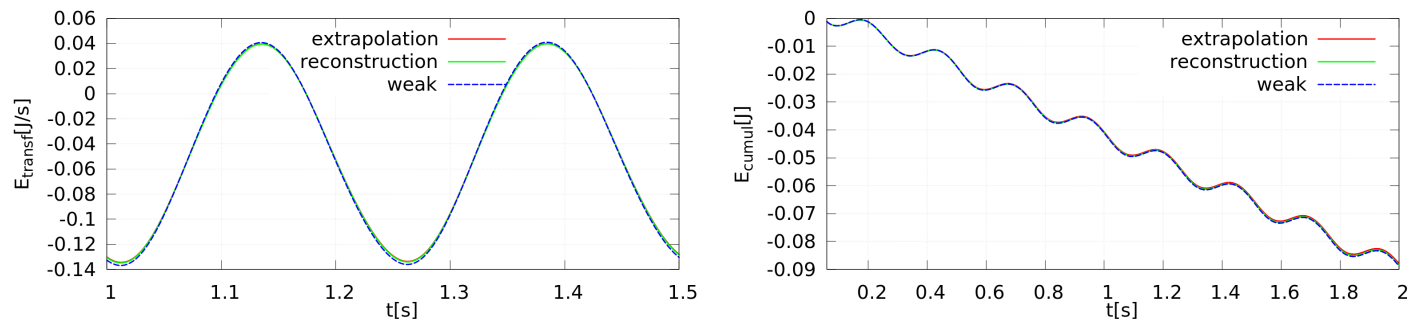

Figure 4: Energy transfer and cumulative functions for cases EX, LR, WF on left, resp. on right.

\section{Conclusion}

Three possible methods of aerodynamic forces calculation (AFC) are presented and their convergence on the simple benchmark of cylinder in cross-flow are studied. The extrapolation from the interior of the fluid domain (EX) is the least accurate method, the local reconstruction (LR) method based on the idea of the local velocity gradient reconstruction by the least square method improves the accuracy compared to EX method. The weak reformulation (WF) method achieves the highest experimental order of convergence (EOC) for the drag and lift coefficients, reaching $E O C \approx 2.5$ for P1-bubble/P1 FE. The weak form of the fluid flow problem and the Green theorem is utilized for the AFC in this case. The implementation effort needed for the LR method is comparable with the WF method.

Further, the static benchmark test is extended to the dynamic one by the prescribed cylinder motion. The EX method reaches in general the lowest values of the (dynamic) drag coefficient as well as the transferred energy whereas the WF method the highest ones what is in correspondence with the previous static results. The differences are relatively small having in maximum incomplete $2 \%$ (for $\nu^{f}=10^{-3} \mathrm{~m}^{2} / \mathrm{s}$ ). Thus we consider all presented methods comparable for the numerical solution of relatively weakly coupled FSI problems. An open question remains if it holds also for strongly coupled cases where a small (and consistent) change in AFC can possibly lead to a different FSI behaviour and where differences in the transferred energy over longer time intervals can become larger.

\section{Acknowledgment}

The financial support for the present project was provided by the Grant No. SGS19/154/OHK2/3T/12 of the Grant Agency of the CTU in Prague and by the ESIF, EU Operational Programme Research, Development and Education, and from the Center of Advanced Aerospace Technology (CZ.02.1.01/0.0/0.0/16_019/0000826), Faculty of Mechanical Engineering, CTU in Prague.

\section{References}

[1] I. Babuška, T. Strouboulis, S.K. Gangaraj, and C.S. Upadhyay: Validation of recipes for the recovery of stresses and derivatives by a computer-based approach. Mathematical and computer modelling, 20(6):45-89, 1994.

[2] M. Braack and P. B. Mucha: Directional do-nothing condition for the Navier-Stokes equations. Journal of Computational Mathematics, 32:507-521, 2014.

[3] R. Clark and E. H. Dowell: A modern course in aeroelasticity. Springer, 2004. 
[4] M. Feistauer, P. Sváček, and J. Horáček: Numerical simulation of fluid-structure interaction problems with applications to flow in vocal folds. In T. Bodnár, G. P. Galdi, and S. Nečasová, editors, Fluid-structure Interaction and Biomedical Applications, pages 312-393. Birkhauser, 2014.

[5] P.M. Gresho and R.L. Sani: Incompressible flow and the finite element method. Volume 1: Advection-diffusion and isothermal laminar flow. Wiley, 1998.

[6] V. John, M. Tabata, and L. Tobiska: Error estimates for nonconforming finite element approximations of drag and lift in channel flows. Univ. Magdenburg, Fak. für Mathematik, 1998.

[7] M. Schäfer, S. Turek, F. Durst, E. Krause, and R. Rannacher: Benchmark Computations of Laminar Flow Around a Cylinder, pages 547-566. Vieweg+Teubner Verlag, 1996.

[8] M. Tabata and K. Itakura: A precise computation of drag coefficients of a sphere. International Journal of Computational Fluid Dynamics, 9(3-4):303-311, 1998.

[9] J. Valášek, P. Sváček, and J. Horáček: On suitable inlet boundary conditions for fluidstructure interaction problems in a channel. Applications of Mathematics, 64(2):225-251, 2019.

[10] J. Valášek, P. Sváček, and J. Horáček: Aerodynamic transfer of energy to vibrating vocal folds for different driving mechanisms. In David Šimurda and Tomáš Bodnár, editors, Topical problems of fluid mechanics 2019, pages 197-204. Institute of Thermomechanics, AS CR, 2019. 